\title{
BM@N and MPD experiments at NICA
}

\author{
Vladimir Kekelidze $^{1, \star}$, Vadim Kolesnikov ${ }^{1}$, and Alexander Sorin ${ }^{1,2}$ \\ ${ }^{1}$ Joint Institute for Nuclear Research, 141980 Dubna, Russia \\ ${ }^{2}$ National Research Nuclear University (MEPhl), 115409 Moscow, Russia
}

\begin{abstract}
The project NICA (Nuclotron-based Ion Collider fAcility) aims to study hot and baryon rich QCD matter in heavy ion collisions in the energy range $\sqrt{s_{N N}}=$ $4-11 \mathrm{GeV}$. The rich heavy-ion physics program will be performed at two experiments, BM@N (Baryonic Matter at Nuclotron) at beams extracted from the Nuclotron, and at MPD (Multi-Purpose Detector) at the NICA collider. This program covers a variety of phenomena in strongly interacting matter of the highest baryonic density, which includes study of collective effects, production of hyperon and hypernuclei, in-medium modification of meson properties, and event-by-event fluctuations.
\end{abstract}

\section{Introduction}

QCD shows the explicit triumph of quantum field theory after the discovery of asymptotic freedom, which makes this theory self-consistent up to the arbitrary small scale at the order of quarks and gluons. In the non-perturbative regime, however, the theory of strong interactions is far from being complete and the study of heavy-ion collisions can shed light on the most fundamental QCD features: confinement and chiral symmetry breaking. Special interest in the study of the QCD phase diagram is related to the region of high baryon density, which is poorly known. In particular, it is of fundamental interest to establish the nature of the deconfinement phase transition and unveil the main features of the QCD diagram, such as the location of the critical endpoint (CEP). One of the main goals of the project Nuclotron-based Ion Collider fAcility (NICA), which is currently under development at the Joint Institute for Nuclear Research (JINR) in Dubna, is to study hot and baryon rich QCD matter in heavy ion collisions in the energy range $\sqrt{s_{N N}}=4-11 \mathrm{GeV}$ [1-4]. Main objects of the NICA facility are presented in Fig. 1. The Nuclotron accelerator with the circumference of $251 \mathrm{~m}$ was put in operation in 1993 and essentially upgraded in 2010-2015. The Nuclotron is designed to provide beams of heavy ions with the kinetic energy up to $4.5 \mathrm{GeV} / \mathrm{n}$ and protons with the kinetic energy up to $12 \mathrm{GeV}$. Beams are extracted to the experimental hall where the first experiment at NICA - Baryonic Matter at Nucloton $(\mathrm{BM} @ \mathrm{~N})$ is allocated. The accelerator development plan includes the construction of the synchrotron Booster and two collider storage rings with the circumference of $503 \mathrm{~m}$, and with two interaction points (IP) for allocation of the Multi-Purpose Detector (MPD) and the Spin Physics Detector (SPD). The NICA collider will provide interactions of gold ions up to center-of-mass energy of $11 \mathrm{~A} \mathrm{GeV}$ with the luminosity of $10^{27} \mathrm{~cm}^{-2} \mathrm{~s}^{-1}$.

\footnotetext{
^e-mail: kekelidze@jinr.ru
} 


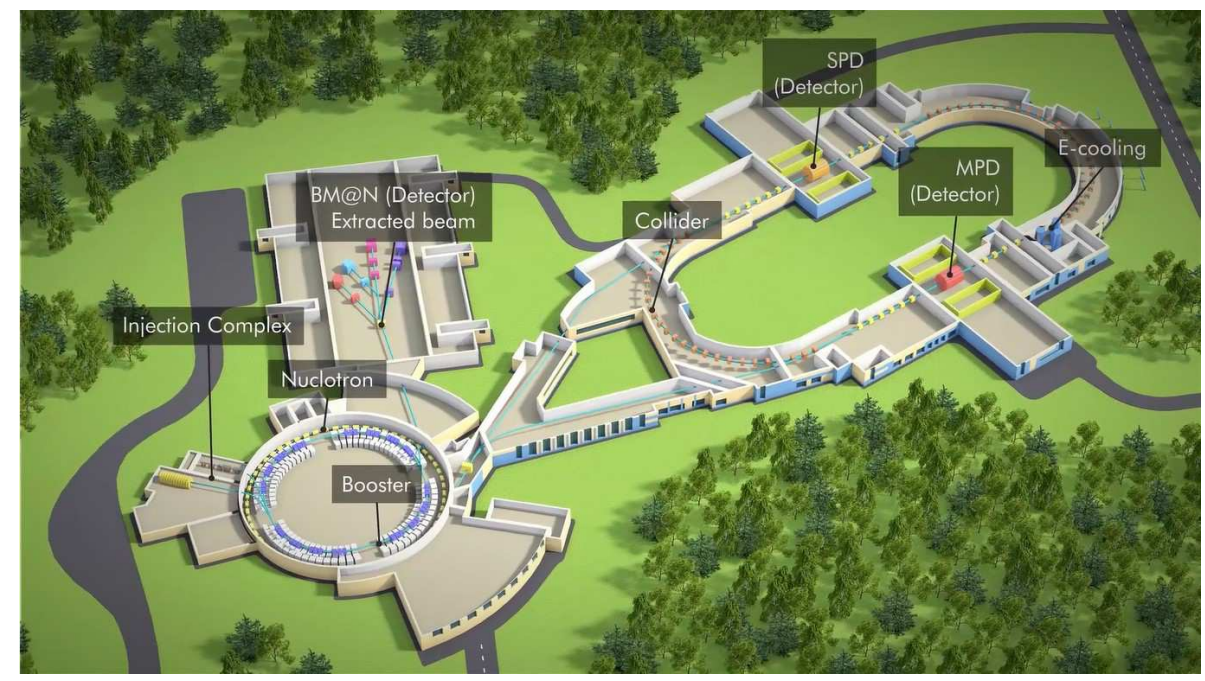

Figure 1. Main objects of the NICA facility; accelerator block: Nuclotron, Booster, Collider; experimental set-ups: BM@N, MPD, and SPD.
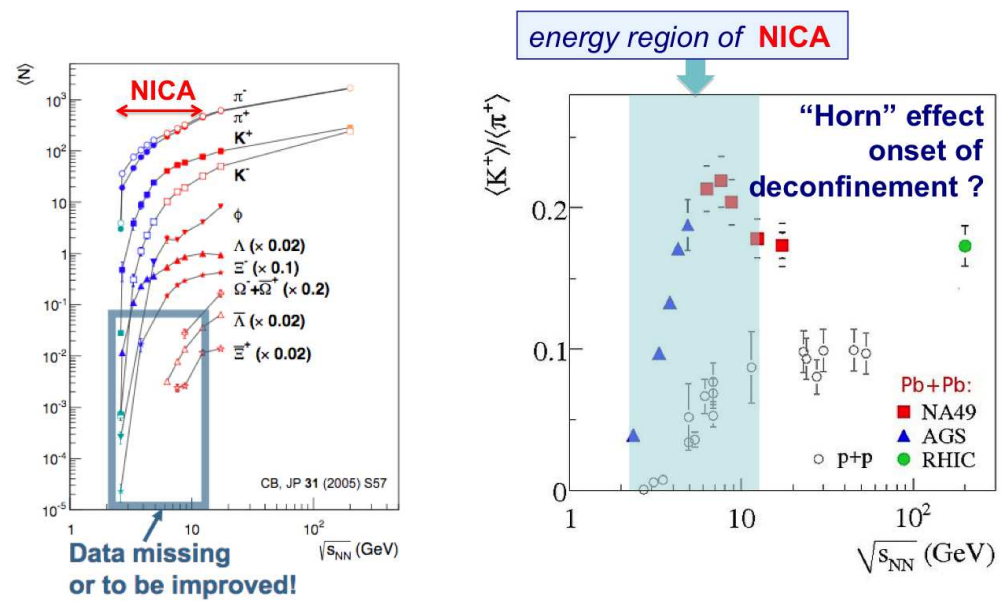

Figure 2. Left: energy dependence of strange particle production; right: excitation function of the $K^{+} / \pi^{+}$ratio.

Production of strange particles in A+A collisions is of particular interest because their enhanced yields (relative to pp reactions) have been predicted as the signal of the deconfinement phase transition. Such enhancement, indeed, was observed at SPS energies [5] and then confirmed at the RHIC. However, the data on strangeness production at energy region of the maximum baryonic density are not complete or even missing (see Fig. 2, left). Recently, both the NA49 and the STAR experiments reported that the ratio of $K^{+}$over $\pi^{+}$has the maximum around $8 \mathrm{GeV} / \mathrm{n}$ in the center-of-mass (see Fig. 2, right). The observed non-monotonic excitation function of the strangeness-to-entropy ratio (Horn) was interpreted as the onset of deconfinement at SPS energies [6]. An alternative explanation 

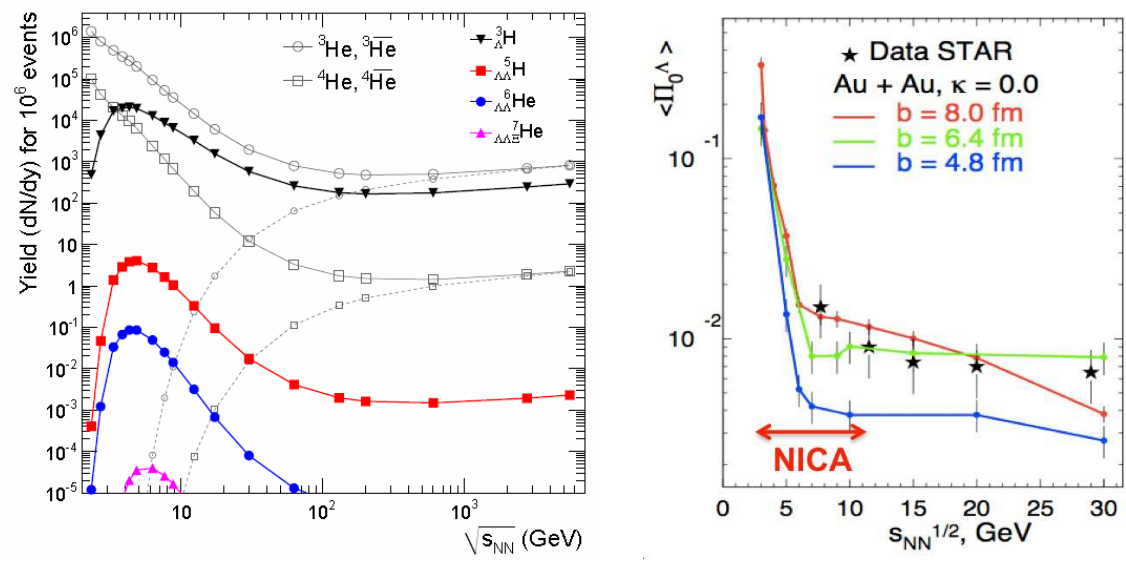

Figure 3. Left: energy dependence of hypernuclei yields in heavy ion collisions calculated in [9]; right: energy dependence of $\Lambda$ polarization in heavy ion collisions.

of the Horn structure in $K / \pi$-ratio was given in Ref. [7] as a result of partial restoration of chiral symmetry (CSR) in hadronic systems at high temperatures and densities. In order to prove or rule out the strangeness enhancement as QGP or CSR indicator more experimental data on strange particle spectra and yields as a function of the system size and centrality are needed. NICA could provide corresponding investigations in the region of the maximum baryonic density, - such maximum for equilibrated states was indeed predicted using thermal model calculations within the NICA energy range [8].

If the deconfinement phase transition in dense hadronic matter is accomplished by partial restoration of the chiral symmetry, the vector meson spectral functions can be modified. Electron-positron pairs (dileptons) from vector meson decays are in this case the best candidates to study such inmedium modifications since they interact only weakly and escape easily having no subsequent strong interactions in the nuclear medium. There is no data on dilepton spectra at center-of-mass energies of several $\mathrm{GeV}$, thus the dilepton program at NICA aims to close this gap.

Hypernuclei provide unique opportunity to study hyperon-nucleus interactions in a many-body environment. Details of the strange sector in the nuclear matter equation-of-state (EOS) are of great importance for astrophysics and should help understanding the mechanism of creation and evolution of super-dense stellar objects - neutron stars. As results of model calculations [9] indicate, the yields of hypernulei in central heavy ion collisions are enhanced within the NICA energy range (see Fig. 3, left), this gives good perspectives for such kind of studies at NICA.

Non-zero polarization of the produced in heavy ion collisions $\Lambda$-hyperons due to so-called vorticity effect was predicted in $[10,11]$. This polarization is related to the anomalously induced axial current, which is proportional to the square of the chemical potential in the system. Calculations indicate that the polarization of Lambda is rising dramatically with the decreasing energy towards the maximum value within the NICA energy range (see Fig. 3, right). Recent STAR BES results reproduce the predicted trend at high collision energies [12] and the studies at lower energies will be continued at NICA.

A landscape of heavy ion collision experiments in the energy region of the maximum baryonic density, including present and future programs, is presented in Fig. 4. Two NICA experiments BM@N with a fixed target and MPD at the collider will cover the whole indicated energy region. 


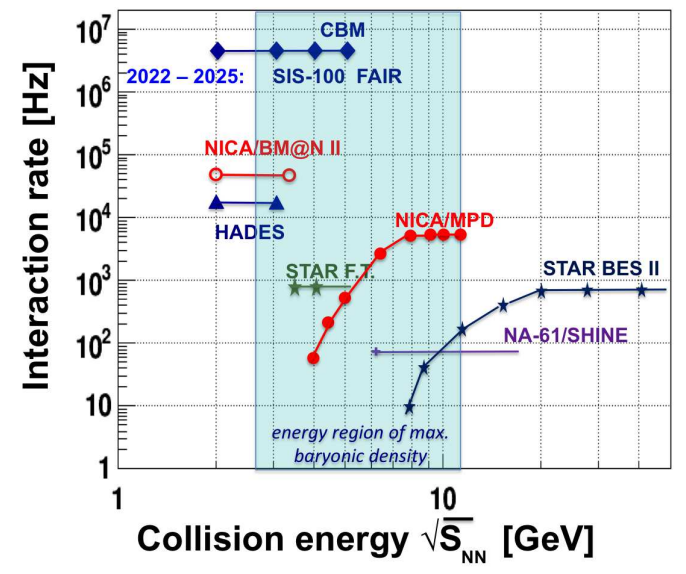

Figure 4. Present and future experiments with heavy ion collisions in the energy range of the maximum baryonic density.

Among the fixed target experiments, CBM at FAIR will provide the largest interaction rate. There are only two collider experiments - NICA and STAR-BES which differ in their interaction rates by 2-3 orders of the magnitude.

Thus, the NICA energy domain has several intriguing features, namely: the transition from baryon dominated matter to meson dominated one; the highest net baryon density - density frontier; it covers the onset of deconfinement region having the maximum in the $K^{+} / \pi^{+}$and $\Lambda / \pi$ ratios, as well as in the $\Lambda$ polarization; a hypothetical 1-st order transition and creation of the mixed phase can be accomplished by the presence of the critical endpoint within the range.

\section{Baryonic Matter at Nuclotron (BM@N)}

A schematic view of the BM@N set-up is shown in Fig. 5. The tracking system is based on 12 planes of GEM detectors installed inside the gap of the dipole magnet of $0.8 \mathrm{~T}$ (SP-41) and drift/pad detectors (DCH, CPC) situated outside the magnet. The fully equipped set-up contains two stations of the Time of Flight (TOF) system, a Zero Degree Calorimeter (ZDC), a Recoil detector, and 4 stations of a Silicon Tracker (ST) installed just downstream the target. Several milestones are foreseen to put in operation the fully equipped detector. The first physical run is planed before the end of 2017 with about a half of the GEM chambers installed, and the complete design configuration is foreseen to be operational in 2020. The BM@N research program is aimed at studying elementary and heavy ion collision with the beam kinetic energy covering the region from 3.5 to $4.5 \mathrm{GeV} / \mathrm{n}$. The results of extensive feasibility studies indicate a good performance of the detector in reconstruction of cascades and hypernuclei (see Fig. 6) with a monthly rate of about $10^{7}$ for each species.

\section{Multi-Purpose Detector (MPD)}

Study of heavy-ion collisions within the energy range of maximum net baryonic density at the NICA collider will be performed with the MPD detector (see Fig. 7) designed as a large uniform acceptance spectrometer for detecting hadrons, electrons and gammas. 


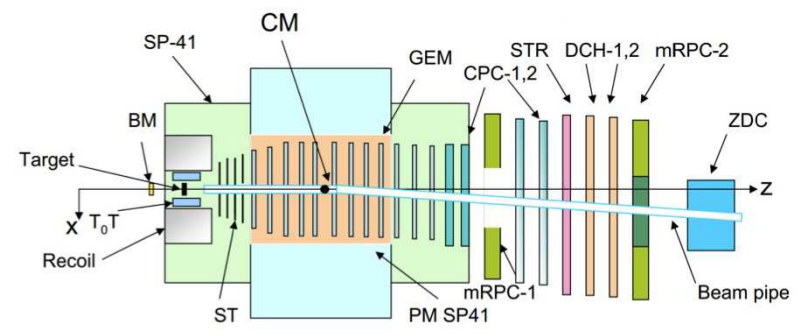

Figure 5. Scheme of the BM@N experiment.
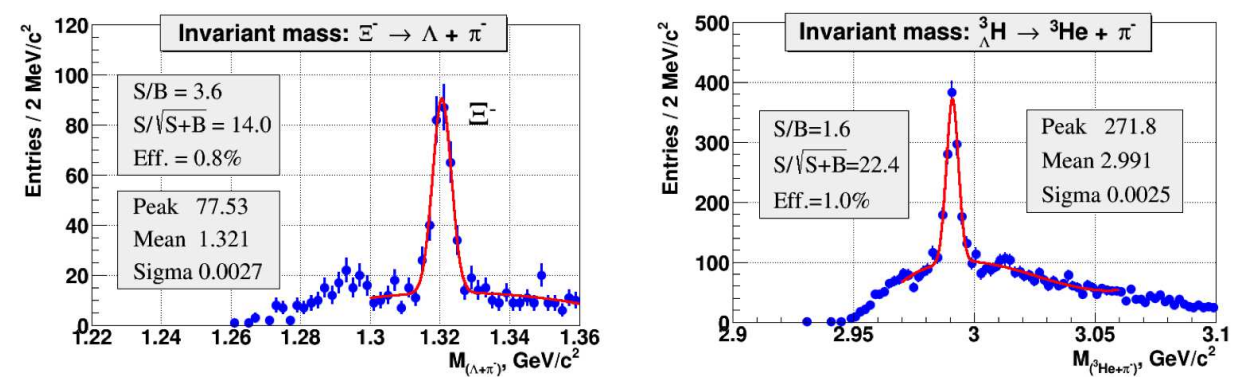

Figure 6. Simulation of reconstructed in BM@N decays of $\Xi^{-}$into $\Lambda$ and $\pi^{-}$(left panel), and decays of hypertriton into ${ }^{3} \mathrm{He}$ and $\pi^{-}$(right panel) for central $\mathrm{Au}+\mathrm{Au}$ collisions at $4.5 \mathrm{~A} \mathrm{GeV}$.

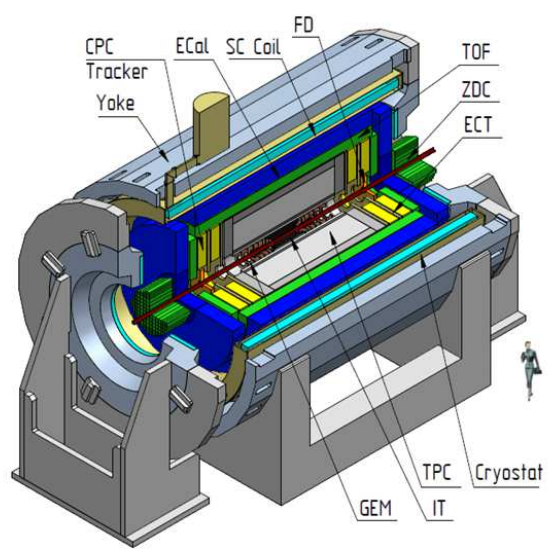

Figure 7. A view of the MPD detector at NICA.

All sub-detectors are placed in a large superconducting solenoid magnet generating the magnetic field of up to $0.5 \mathrm{~T}$. The magnetic field inhomogeneity inside the tracking volume will be less than $10^{-3}$ in order to ensure a good tracking performance. The main tracking device of the MPD detector - the cylindrical Time-Projection Chamber (TPC) of $2.8 \mathrm{~m}$ (diameter) by $3.4 \mathrm{~m}$ (length) will provide trajectory reconstruction within the pseudorapidity range $|\eta|<2$. Signals are readout with wire cham- 

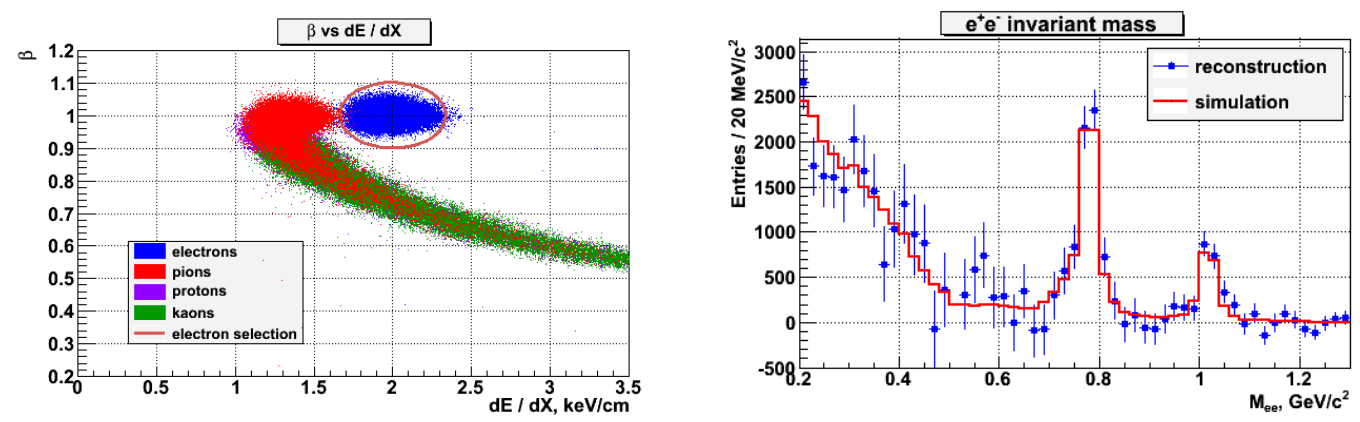

Figure 8. Left: correlation of the $d E / d x$ and TOF information in MPD, the red line indicates the PID ellipse for electrons; Right: background-subtracted invariant mass distributions of electron-positron pairs from central $\mathrm{Au}+\mathrm{Au}$ collisions at MPD.

bers (98000 channels in total) located at the both end faces. As an alternative read out technology for an upgraded TPC version GEM detectors are considered. For the midrapidity tracks the TPC will provide up to 53 measurements of the specific ionization and thus allow particle identification (PID) via energy loss $(d E / d x)$ measurements with the precision better than $8 \%$. The TOF system, which provides an extra PID power for charged tracks, consists of a barrel and two endcap systems equipped with Multigap Resistive Plate Chambers (MRPC). Having the intrinsic time resolution better than $70 \mathrm{ps}$ the TOF system will allow hadron discrimination up to several $\mathrm{GeV} / \mathrm{c}$ momenta. Two arrays of quartz counters (FD) located at a distance of $140 \mathrm{~cm}$ at each side from the MPD center will provide fast timing and triggering for the experiment. The Electromagnetic Calorimeter (ECAL) for electron and gamma measurements located behind the TOF detector will be built of lead-scintillator sampling modules based on the "shashlyk" technology. ECAL comprises about 43000 modules of 18 radiation length thickness and of about $3 \mathrm{~cm}^{2}$ cross section forming a barrel shape with projective geometry directing to the IP. The MPD two-arm hadron calorimeter FHCAL, which is made of 44 modules with transverse dimensions of $15 \times 15 \mathrm{~cm}^{2}$, is planned to be used for event plane reconstruction and definition of the reaction centrality. The FHCAL design covering the pseudorapidity range $2.0<|\eta|<4.8$ allows measuring the reaction plane with the accuracy of $\approx 20^{\circ}$ and to define centrality with a better then $10 \%$ precision. The inner tracker (IT) system maximizing the MPD performance for vertex reconstruction will be based on MAPS technology developed by the ALICE experiment.

The MPD detector will be put in operation in two stages. At the first stage MPD will be equipped with the TPC and barrel parts of the TOF and ECAL systems. The FHCal calorimeter and the FD detector will be operational as well. At the second stage MPD will be supplemented with the IT system and the endcap sub-detectors (the Straw tube tracker complemented by the endcap parts of the TOF and ECal systems).

The dedicated Monte-Carlo feasibility studies, including event generation, particle transport and realistic detector simulation, were carried out to investigate MPD performance during the first stage of the NICA physics program realization. Figure 8 (left panel) indicates the reliable electron identification using the combined information from the TOF and TPC detectors. Additional information about the energy deposit from the ECAL calorimeter allows reducing the hadron contamination in the electron sample down to the level of $10^{-5}$. That could provide the reliable vector meson reconstruction though their dilepton decays in heavy-ion collisions at MPD (see Fig. 8, right). 

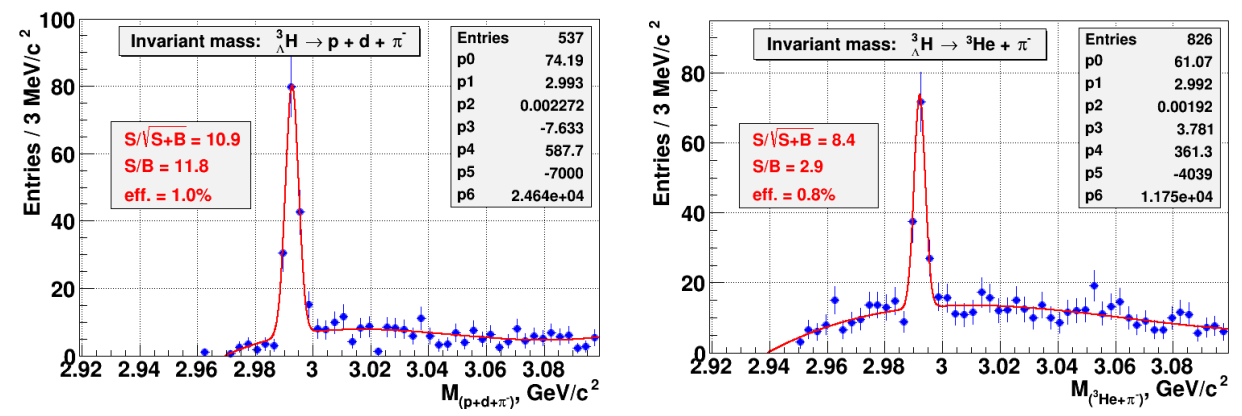

Figure 9. Reconstruction of two modes of hypertriton decays into $p+d+\pi^{-}$(left) and ${ }^{3} \mathrm{He}+\pi^{-}$(right).
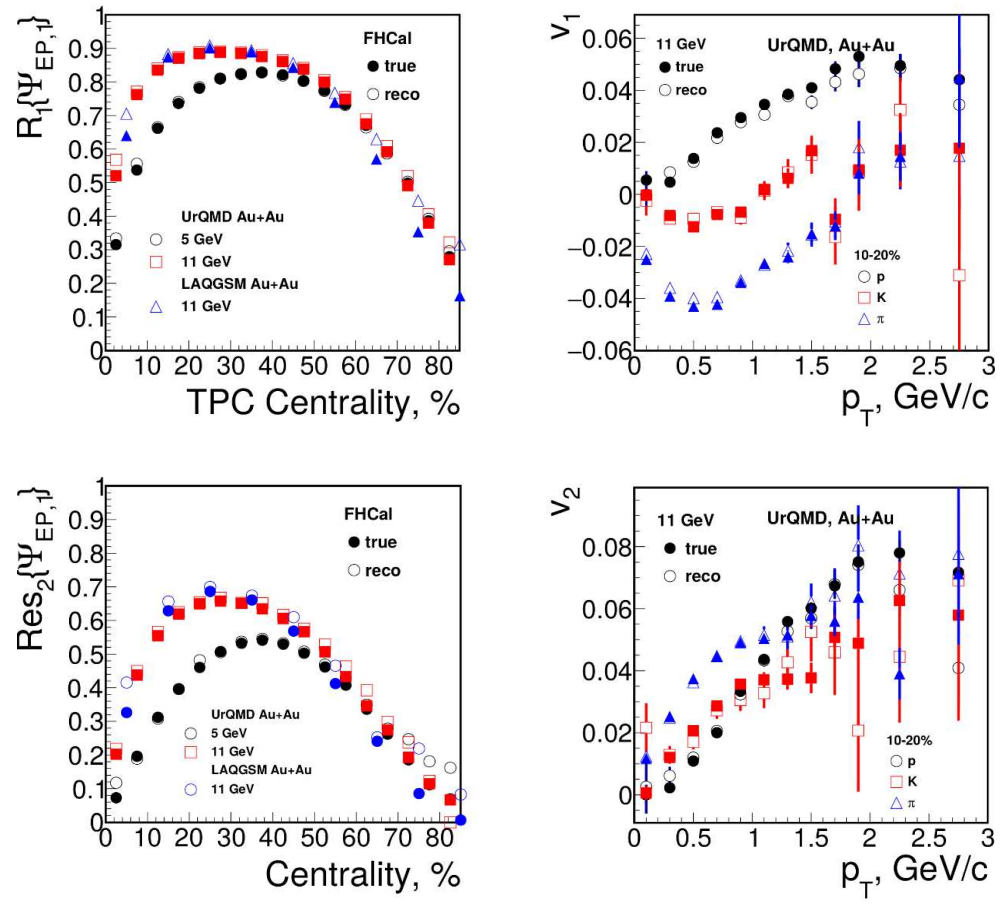

Figure 10. Correction factors of reaction plane reconstructions for the first (upper, left) and second (down, left) harmonics of flow Fourier decompositions; reconstructed first (upper, left) and second (down, right) harmonics of flows versus $p_{t}$.

An analysis of the hypernuclei reconstruction has been performed with $5 \cdot 10^{5}$ central $\mathrm{Au}+\mathrm{Au}$ events, which corresponds to about 30 minutes of data taking time at NICA. The obtained invariant mass spectra for reconstruction of two modes of simulated hypertriton decays are presented in Fig. 9.

MPD ability to measure azimuthal asymmetry in heavy ion collisions is presented in Fig. 10. Centrality of reaction was defined by means of TPC track multiplicity, and the reaction plane was 
reconstructed using the FHCal responses. The correction factors $R_{1,2}$ for the first and second harmonics of flow decomposition are presented in the left panels, while the reconstructed first $\left(v_{1}\right)$ and second $\left(v_{2}\right)$ flow coefficients for various particles are presented on the right panels as a function of $p_{t}$. The presented results indicate good precisions of reaction plane reconstruction and sufficiently good reproducibility of the generated events in a wide range of transverse momentum.

\section{Conclusions and perspectives}

The project NICA, which is aimed at the construction of a new accelerator facility for heavy ions and polarized beams, is under development at JINR (Dubna). NICA ambitious goals cover many domains including experimental exploration of the QCD phase diagram with heavy ions, spin program with an emphasis on the nucleon spin structure, accelerator R\&D, and intensive infrastructure upgrades. The project realization plan foresees a staged construction of the accelerator complex with the booster commissioning getting started by the end of 2018 as the first milestone. The start-up configuration of the NICA collider will be ready by the end of 2020, and its design high luminosity parameters are foreseen for 2023. The construction of the experimental setups at NICA is going close to the schedule. The BM@N experiment starts taking data in 2017 with a half of the tracking and PID systems installed, while its complete configuration will be ready in 2020. The first stage of the MPD detector for study nucleus-nucleus collisions at midrapidity will be ready in 2020 and further upgraded in 2023 to cover close to the full phase space.

The NICA project is open and attractive to a wide scientific community, its international collaborations currently involve more than 70 institutions from 25 countries. The future scientific infrastructure of the NICA facility will be able to provide research opportunities for about 1000 visitors, scientists and engineers working in accelerator physics, particle and nuclear physics, as well as in applied research areas, including material science and medicine. Beams of the NICA complex will be used not only for fundamental research but also for innovation and technological activities.

Realization of the NICA project, which has been included in the 2016 issue of the road-map of European Strategy Forum of Research Infrastructure (ESFRI), in the list of research infrastructures of global interest of Group of Senior Officials (GSO) on Global Research Infrastructures, and NuPECC Long Range Plan 2017, will provide new opportunities for the experimental exploration of nuclear matter of the highest baryonic density.

\section{References}

[1] Kekelidze V.D. et al. (NICA and MPD Collab.), Phys. Atom. Nucl. 75 542-545 (2012).

[2] Kekelidze V.D. et al. (NICA Collab.), EPJ Web Conf. 9501014 (2015).

[3] Kekelidze V.D. et al. (NICA Collab.) Nucl. Part. Phys. Proc. 273-275 170-174 (2016).

[4] Kekelidze V.D. et al. (NICA Collab.) JINST 12 No.06 C06012 (2017).

[5] Antinori F. et al. (NA57 Collab.) J. Phys. G. 32, 427 (2006).

[6] Alt C. et al. (NA49 Collab.) Phys. Rev. C 77, 024903 (2008).

[7] W. Cassing, A. Palmese, P. Moreau, E. Bratkovskaya Phys. Rev. C 93, 014902 (2016).

[8] Randrup J., Cleymans J. Phys. Rev. C 74, 047901 (2006).

[9] Andronic A., Braun-Munzinger P., Stachel J., Stocker H., Phys. Lett. B 697, 203 (2011).

[10] Rogachevsky O., Sorin A., Teryaev O. Phys. Rev. C 82, 054910 (2010).

[11] Baznat M., Gudima K., Sorin A., Teryaev O. E-print: arXiv:1701.00923.

[12] Adamczyk L. et al. (STAR Collab.), Nature 548, 62-65 (2017). 